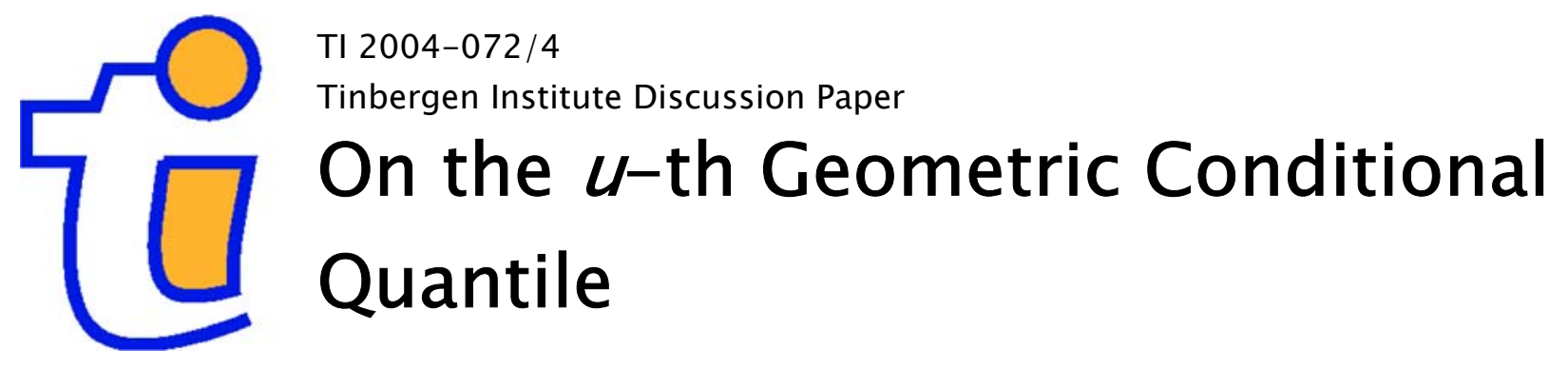

\author{
Yebin Cheng \\ Jan G. de Gooijer
}

Department of Quantitative Economics, Faculty of Economics and Econometrics, Universiteit van Amsterdam, and Tinbergen Institute. 


\section{Tinbergen Institute}

The Tinbergen Institute is the institute for economic research of the Erasmus Universiteit Rotterdam, Universiteit van Amsterdam, and Vrije Universiteit Amsterdam.

Tinbergen Institute Amsterdam

Roetersstraat 31

1018 WB Amsterdam

The Netherlands

Tel.: $\quad+31(0) 205513500$

Fax: $\quad+31(0) 205513555$

Tinbergen Institute Rotterdam

Burg. Oudlaan 50

3062 PA Rotterdam

The Netherlands

Tel.: $\quad+31(0) 104088900$

Fax: $\quad+31(0) 104089031$

Please send questions and/or remarks of nonscientific nature to driessen@tinbergen.nl.

Most TI discussion papers can be downloaded at http://www.tinbergen.nl. 


\title{
On the $u$-th Geometric Conditional Quantile
}

\author{
Yebin Cheng ${ }^{1}$ and Jan G. De Gooijer ${ }^{2 *}$ \\ ${ }^{1}$ Tinbergen Institute and Department of Quantitative Economics, \\ University of Amsterdam, \\ Roetersstraat 11, 1018 WB Amsterdam, The Netherlands \\ Telephone: +31-20-525 7110; Fax: +31-20-525 4349 \\ e-mail: y.cheng@uva.nl \\ 2 Department of Quantitative Economics, University of Amsterdam, \\ Roetersstraat 11, 1018 WB Amsterdam, The Netherlands \\ Telephone: +31-20-525 4244; Fax: +31-20-525 4349 \\ e-mail: j.g.degooijer@uva.nl
}

\begin{abstract}
Motivated by Chaudhuri's work (1996) on unconditional geometric quantiles, we explore the asymptotic properties of sample geometric conditional quantiles, defined through kernel functions, in high dimensional spaces. We establish a Bahadur type linear representation for the geometric conditional quantile estimator and obtain the convergence rate for the corresponding remainder term. From this, asymptotic normality on the estimated geometric conditional quantile is derived. Based on these results we propose confidence ellipsoids for multivariate conditional quantiles. The methodology is illustrated via data analysis and a Monte Carlo study.
\end{abstract}

AMS 2000 subject classification: $62 \mathrm{G} 20,62 \mathrm{G} 05$.

Key words and phrases: Asymptotic normality; Bahadur representation; geometric conditional quantile; confidence ellipsoids; kernel function.

${ }^{*}$ Corresponding author 


\section{Introduction}

Researchers are often interested in how an $s$-dimensional covariate $X$ affects a $d$-dimensional response variable $Y$, using a sample of independent and identically distributed data pairs $\left\{\left(X_{1}, Y_{1}\right), \ldots,\left(X_{n}, Y_{n}\right)\right\}(n \geq d+s)$. Conditional quantiles are a key aspect of this problem. When the functional form is unknown a wide variety of techniques are available to estimate univariate conditional quantiles, including kernel and nearest neighbor approaches, quantile regression, and smoothing splines. In contrast, relative little attention has been paid to multivariate conditional quantiles and their sample properties. The problem was recently tackled by De Gooijer, Gannoun, and Zerom (2002) via generalizing the notion of multivariate unconditional quantiles, based on norm minimization, given by Abdous and Theodorescu (1992).

Here we focus on an alternative formulation of multivariate conditional quantiles generalizing a notion of geometric or spatial quantile studied by Chaudhuri $(1992,1996)$. Chaudhuri indexed multivariate unconditional quantiles, based on the Euclidean distance, by the elements of a $d$ dimensional open unit ball. The corresponding quantiles not only give the idea of "extreme" or "central" observations but also about their orientations in a multivariate data cloud. The basic idea of both the Abdous-Theodorescu approach, and the Chaudhuri approach comes from the well-known fact that for univariate $Y$ with $\mathbb{E}|Y|<\infty$, the $p$-th quantile for $0<p<1$ may be characterized as any value $\theta$ minimizing $\mathbb{E}\{|Y-\theta|+(2 p-1)(Y-\theta)\}$; see Serfling (2002) for a detailed comparison of the two approaches. Chakraborty (2003) also investigated a similar problem but for multiresponse linear regression models.

When $d \geq 2$, it is relatively straightforward to extend the geometric quantile to the geometric conditional quantile. For any vector $u=\left(u_{1}, \ldots, u_{d}\right)^{T}$ in the open unit ball $B^{(d)}=$ $\left\{u \mid u \in \mathbb{R}^{d},\|u\|<1\right\}$ and any $t \in \mathbb{R}^{d}$, denote by $\Phi(u, t)=\|t\|+\langle u, t\rangle$, where $\|\cdot\|$ is the usual Euclidean norm and $\langle\cdot, \cdot\rangle$ is the usual Euclidean inner product. Then, the $u$-th geometric conditional quantile of $Y$ given $X=x$ is defined as

$$
Q(u \mid x)=\arg \min _{\theta \in \mathbb{R}^{d}} \mathbb{E}\{\Phi(u, Y-\theta)-\Phi(u, Y) \mid X=x\} .
$$

The estimate of the geometric conditional quantile $Q(u \mid x)$ is defined as follows through the empirical distribution. As usual, the nonparametric estimate of the conditional distribution $F(y \mid x)$ is given by the so-called Nadaraya-Watson estimate as $F_{n}(y \mid x)=\sum_{i=1}^{n} w_{n, i} \mathbb{I}_{\left(Y_{i} \leq y\right)}$, where the weight functions $w_{n, i}(i=1,2, \ldots, n)$ are given by

$$
w_{n, i}=\frac{K_{h_{n}}\left(x-X_{i}\right)}{\sum_{i=1}^{n} K_{h_{n}}\left(x-X_{i}\right)}
$$


$K_{h_{n}}(\cdot)=\frac{1}{h_{n}^{s}} K\left(\frac{\cdot}{h_{n}}\right), K(\cdot)$ is the kernel function, $h_{n}>0$ is the bandwidth, and $\mathbb{I}_{A}$ denotes the indicator function for set $A$. Then the estimator of the $u$-th geometric conditional quantile $Q(u \mid x)$ is defined subsequently by

$$
\begin{aligned}
\hat{Q}_{n}(u \mid x) & =\arg \min _{\theta \in \mathbb{R}^{d}} \int_{\mathbb{R}^{d}}\{\Phi(u, y-\theta)-\Phi(u, y)\} F_{n}(d y \mid x) \\
& =\arg \min _{\theta \in \mathbb{R}^{d}} \sum_{i=1}^{n} w_{n, i}\left\{\Phi\left(u, Y_{i}-\theta\right)-\Phi\left(u, Y_{i}\right)\right\} .
\end{aligned}
$$

In this paper, we establish a Bahadur type linear representation of the geometric conditional quantile estimator (1.1) and obtain the convergence rate for its corresponding remainder term. From this, the asymptotic normality for the estimator is derived. The method avoids the strong condition imposed by Cadre and Gannoun (2000) in the proof of the asymptotic normality of the conditional $L_{1}$-median estimator. With the asymptotic convergence and distribution we are able to construct confidence ellipsoids for the geometric conditional quantile estimator. Also, on the basis of these results, one can in principle formulate goodness-of-fit statistics and study their properties. Our method of proof is somewhat similar to Chaudhuri's $(1992,1996)$.

The rest of the paper is organized as follows. In Section 2 we present the conditions, under which the main theorems are valid, followed by the statements of the main results. Asymptotic confidence ellipsoids are constructed in Section 3. Further, we conduct a simulation study, to asses the performance of the ellipsoids in finite sample situations. After given an empirical illustration in Section 4, we close with a brief summary in Section 5. All proofs are relegated to the Appendix, including proofs of lemmas which are of some general interest. Throughout the paper, $f(x, y)$ and $g(x)$ denote the density functions of $(X, Y)$ and $X$, respectively. And $f(y \mid x)$ is the conditional density function of $Y$ given $X=x$. The notation $\stackrel{\mathcal{D}}{\rightarrow}$ refers to converge in distribution. $A^{T}$ denotes the transpose of some matrix $A$. Also, we adopt the convention that for $x \in \mathbb{R}^{d}, U(x)=x /\|x\|$ for $x \neq 0$ and $U(x)=0$ for $x=0$. Unless otherwise specified, the limits in this paper are taken as $n \rightarrow \infty$.

\section{Main Result}

Before giving the Bahadur type linear representation and asymptotic normality for $\hat{Q}_{n}(u \mid x)$, we list some required conditions first.

Condition 1. Suppose that for any sufficiently small $\|z\|>0, z \in \mathbb{R}^{s}$, there exists some 
constants $\lambda_{1}, \lambda_{2}>0$ such that

$$
\int|f(x+z, y)-f(x, y)| d y=O\left(\|z\|^{\lambda_{1}}\right)
$$

and

$$
\int \frac{1}{\|y\|}|f(x+z, y)-f(x, y)| d y=O\left(\|z\|^{\lambda_{2}}\right)
$$

Condition 2. i). $f(y \mid x)$ is bounded on every bounded set of $\mathbb{R}^{d}$ and $f(y \mid \cdot)$ is continuous at $x ; \ddot{a}) . g(x)>0$ and $g(\cdot)$ is continuous at the point $x ; \ddot{u})$. the support of $f(y \mid x)$ on $y$ is not included in a straight line.

Condition 3. There exists positive numbers $r, c_{1}$, $c_{2}$ such that $c_{1} I_{(\|x\| \leq r)} \leq K(x) \leq c_{2} I_{(\|x\| \leq r)}$. Condition $4 . h_{n} \rightarrow 0, n h_{n}^{s} \sim n^{\gamma}$ for some constant $0<\gamma<\frac{d \min \left(\lambda_{1}, \lambda_{2}\right)}{(d+1) s+d \min \left(\lambda_{1}, \lambda_{2}\right)}$.

Condition 5. For any $t$ in the neighbor of $x$, the functions $g(t)$ and $\mathbb{E}[U(Y-Q(u \mid x)) \mid X=t]$ on the variable $t$ have the second bounded derivative in the neighbor of $x$. Also, the function

$$
\mathbb{E}\left[(U(Y-Q(u \mid x)+u))(U(Y-Q(u \mid x)+u))^{T} \mid X=t\right]
$$

on $t$ is continuous at the point $x . n h_{n}^{s+4} \rightarrow 0$ and $K(\cdot)$ has the second derivative.

Theorem 2.1. Under Conditions 1 to $4, \hat{Q}_{n}(u \mid x)$ has the following Bahadur type representation

$$
\hat{Q}_{n}(u \mid x)-Q(u \mid x)=D_{1}^{-1} \sum_{i=1}^{n} w_{n, i}\left(U\left(Y_{i}-Q(u \mid x)\right)+u\right)+R_{n}
$$

with probability one, where $D_{1}=\mathbb{E}[P(Y-Q(u \mid x)) \mid X=x]$ and $P(Y)=\frac{1}{\|Y\|}\left(I_{d}-U(Y) U^{T}(Y)\right)$. The remainder term $R_{n}=O\left(\log n /\left(n h_{n}^{s}\right)\right)$ if $d \geq 3$. And it is equal to o $\left(\left(\log n /\left(n h_{n}^{s}\right)\right)^{w}\right)$ for $0<w<1$ if $d=2$.

From Theorem 2.1, Cramér and Wold's Theorem (see Serfling (1986), p. 18), Corollary IV.2 and Theorem II.1 of Bosq and Lecoutre (1987), the following asymptotic normality on $u$-th geometric conditional quantile can be inferred as follows.

Corollary 2.2. Under conditions of Theorem 2.1 and Condition 5, it holds that

$$
\sqrt{\frac{n h_{n}^{s} g_{n}(x)}{\int K^{2}(t) d t}} D_{(n)}^{-\frac{1}{2}} D_{1}\left(\hat{Q}_{n}(u \mid x)-Q(u \mid x)\right) \stackrel{\mathcal{D}}{\rightarrow} N\left(0, I_{d}\right),
$$

where $N\left(0, I_{d}\right)$ is the normal distribution with mean 0 and variance $I_{d}$, which is a $d \times d$ identity matrix, $g_{n}(x)=\frac{1}{n h_{n}^{s}} \sum_{i=1}^{n} K\left(\frac{x-X_{i}}{h_{n}}\right)$ and

$$
D_{(n)}=\sum_{i=1}^{n} w_{n, i}\left(U\left(Y_{i}-\hat{Q}_{n}(u \mid x)\right)+u\right)\left(U\left(Y_{i}-\hat{Q}_{n}(u \mid x)\right)+u\right)^{T} .
$$


Furthermore, if $d=2$ with the condition that $\mathbb{E}\left(\frac{1}{\|Y-Q(u \mid x)\|^{2}}\right)<\infty$ or $d \geq 3$, then (2.3) also holds with $D_{1}$ replaced by

$$
D_{1, n}=\sum_{i=1}^{n} w_{n, i} P\left(Y_{i}-\hat{Q}_{n}(u \mid x)\right) .
$$

In Theorem 2.1 and Corollary 2.2, via Remark 4 and Lemma 5.3 of Chaudhuri (1992), we know that both the matrix $D_{1}$ itself and its inverse matrix exist in view of $d \geq 2$. Because the sequence of random variables $\left\{Y_{i} ; i=1,2, \ldots, n\right\}$ is absolute continuous, it doesn't fall into a straight line in $\mathbb{R}^{d}$ almost surely. Then by the standard arguments on multivariate quantile (see Chaudhuri, 1996) and Theorem 2.17 of Kemperman (1987), we know that the geometric conditional quantile $\hat{Q}_{n}(u \mid x)$ exists uniquely for any $u \in B^{(d)}$. Also, according to Kemperman (1987), the existence and the uniqueness for the $u$-th geometric conditional quantile $Q(u \mid x)$ are judged by $\dddot{m}$ ) of Condition 2. The properties on geometric quantile for the transformed data, as mentioned in Section 2.2 of Chaudhuri (1996), will also hold analogously for geometric conditional quantile.

\section{Simulations}

\subsection{Design}

From Corollary 2.2 it directly follows that the $100(1-\alpha) \%(\alpha \in(0,1))$ asymptotic confidence ellipsoid for $Q(u \mid x)$ is given by

$$
c\left(\hat{Q}_{n}(u \mid x)-Q(u \mid x)\right)^{T} S^{-1}\left(\hat{Q}_{n}(u \mid x)-Q(u \mid x)\right) \leq \chi_{d}^{2}(\alpha)
$$

where $c=n h_{n}^{s} g_{n}(x) / \int K^{2}(t) d t, S^{-1}=D_{1}^{T} D_{(n)}^{-1} D_{1}$ and $\chi_{d}^{2}(\alpha)$ denotes the $100(1-\alpha)$ th percentile of a chi-squared distribution with $d$ degrees of freedom. To assess the performance of (3.1) in finite samples we conduct a Monte Carlo experiment. For visual purposes, we restrict the choice of the dimensions to the case $d=2$ and $s=1$. Let $\rho=\left(\rho_{Y_{1}, Y_{2}}, \rho_{Y_{1}, X}, \rho_{Y_{2}, X}\right)$ be the corresponding set of correlation coefficients. Then, all simulation results reported below will be based on observations $\left(X_{i}, Y_{i}\right)(i=1,2, \ldots, n)$ generated from the trivariate normal distribution $N(0, \Sigma)$ with variance-covariance matrix

$$
\Sigma=\left(\begin{array}{ccc}
1 & \rho_{Y_{1}, Y_{2}} & \rho_{Y_{1}, X} \\
\rho_{Y_{1}, Y_{2}} & 1 & \rho_{Y_{2}, X} \\
\rho_{Y_{1}, X} & \rho_{Y_{2}, X} & 1
\end{array}\right) .
$$


a) $u=(-0.8,-0.58)^{\top} ; \rho=(0.5,0.2,-0.4) ; x=0$

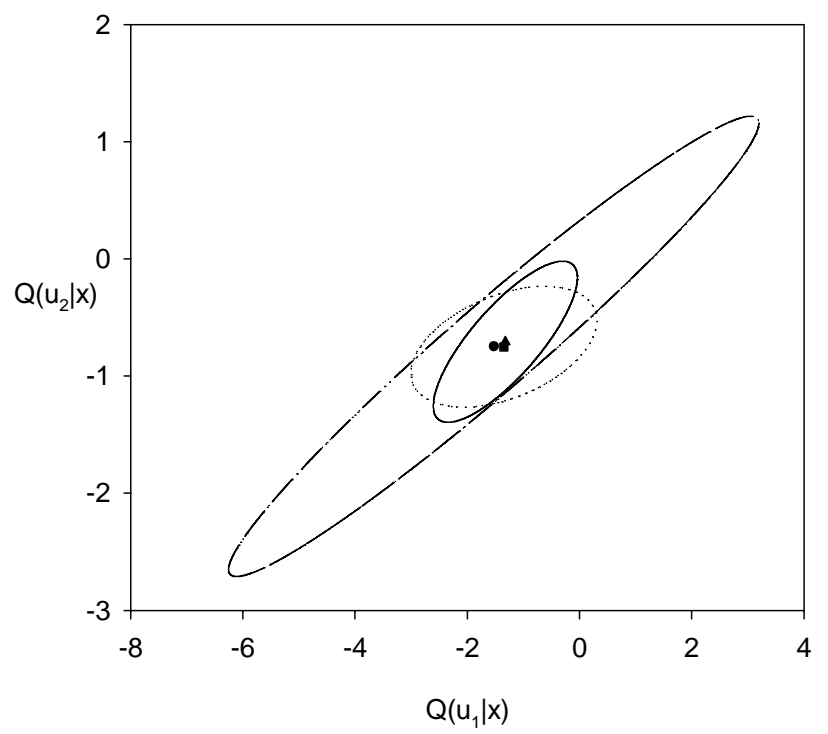

b) $u=(0.8,-0.4)^{\top} ; \rho=(0.5,0.2,0.4) ; x=0$

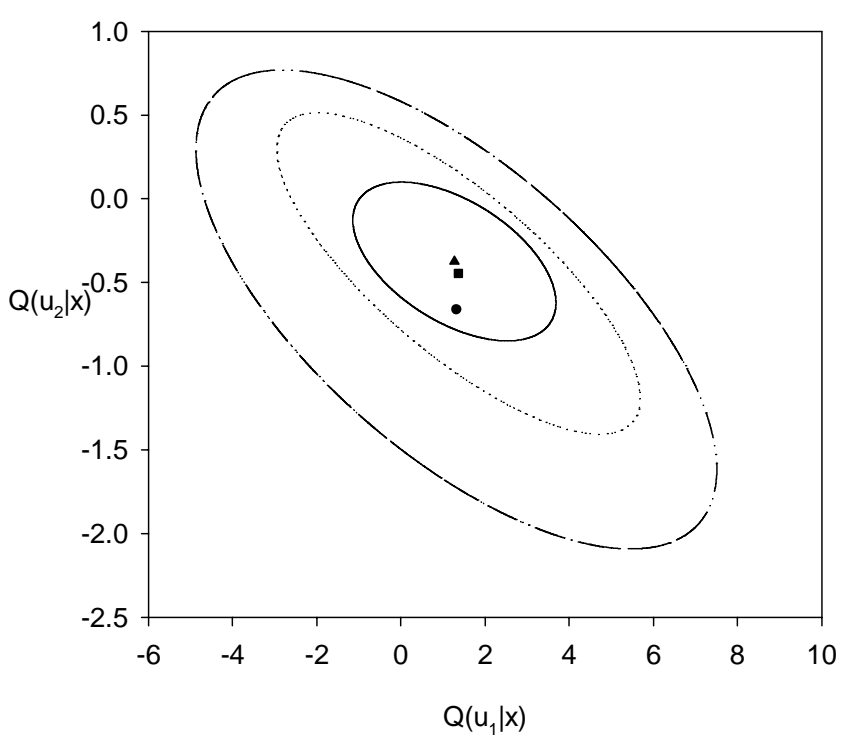

Figure 1: 95\% confidence ellipses for $Q(u \mid x)$ for $n=100$ (dash-dotted-dotted lines), $n=200$ (dotted lines), and $n=500$ (solid lines); the centers of the ellipses at $\left(\hat{Q}_{n}\left(u_{1} \mid x\right), \hat{Q}_{n}\left(u_{2} \mid x\right)\right)$ are denoted by black dots $(n=100)$, black squares $(n=200)$, and black triangles $(n=500)$.

Here, it should be noted that the normal distribution satisfies the conditions of Corollary 2.2 with $\lambda_{1}=\lambda_{2}=1$. For the estimation of $Q(u \mid x)$ we choose the kernel $K(\cdot)$ to be the bivariate standard normal density $N\left(0, I_{2}\right)$, so $\int K^{2}(t) d t=1 / 2(\pi)^{1 / 2}$. The bandwidth $h_{n}$ is set at $n^{\gamma-1}$ with $\gamma=(2 / 5)-0.01$. This choice satisfies Condition 4 .

\subsection{Results}

One way to assess the finite sample performance of (3.1) is to compute the lengths of the major an minor axes of the confidence ellipses. As $n$ increases, we expect from Corollary 2.2 that these lengths will decrease. Setting $u=(-0.8,-0.58)^{T}$, which corresponds to an extreme geometric conditional quantile ( $\|u\|=0.9764)$, Figure 1.a) shows $95 \%$ confidence ellipses for $n=100,200$, and 500 when $\rho=(0.5,0.2,-0.4)$ and $x=0$. Observe that for size $n=500$ the lengths of the principal axes of both ellipses (solid lines) are much shorter than those observed for $n=200$ and $n=100$. Further, the lengths of the axes of the confidence ellipses for $n=100$ (dashdotted-dotted lines) are much shorter than those for $n=200$ (dotted lines). Figures 1.b) shows that this observation also holds in the case $u=(0.8,-0.4)^{T}(\|u\|=0.8), \rho=(0.5,0.2,0.4)$, and $x=0$. Similar Monte Carlo results were obtained for other values of $u$, sample sizes $n$, 


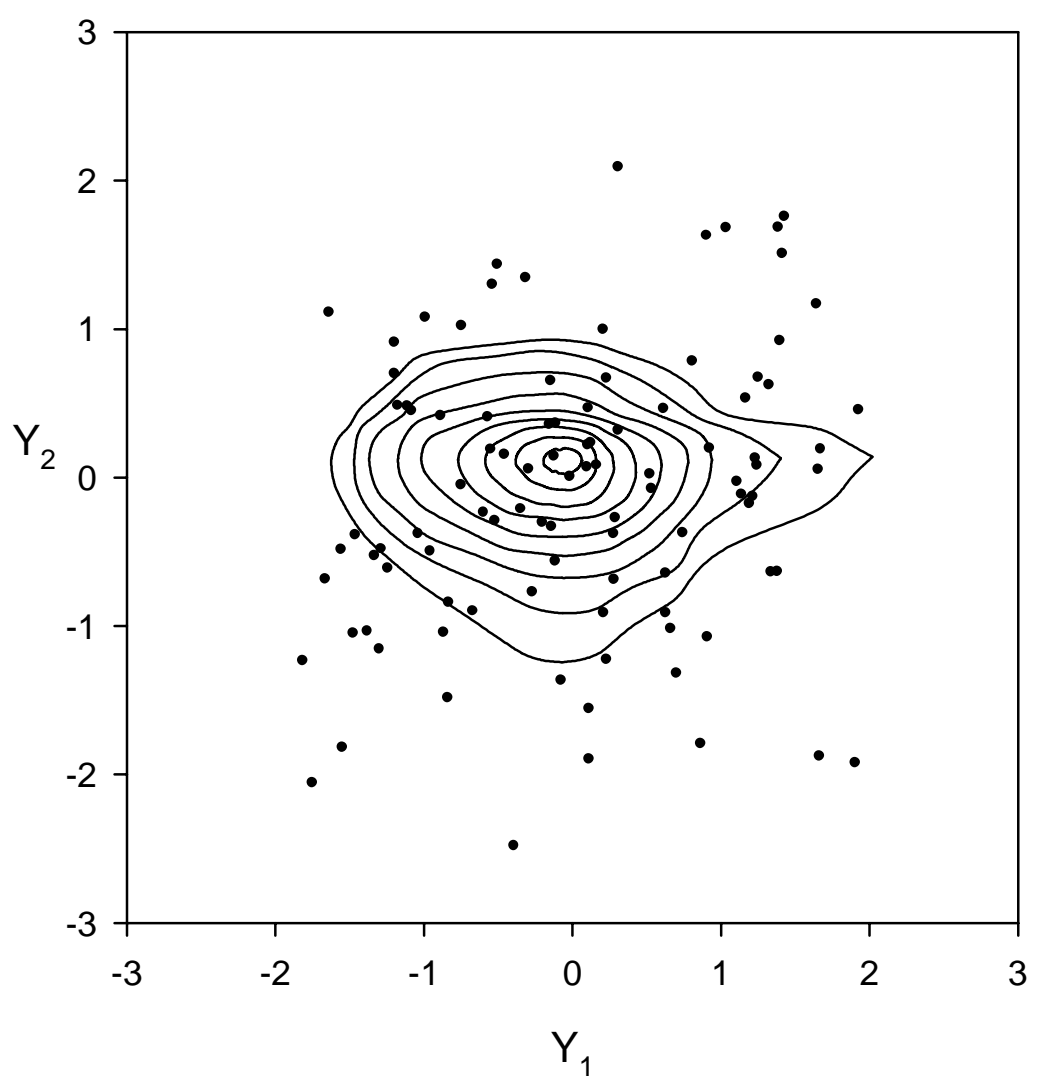

Figure 2: Contour plots of the $u$-th geometric conditional quantile for $u=r(\cos \theta, \sin \theta)^{T}$ with $r=0.1$ (inner contour lines), $0.2, \ldots, 0.9$ (outer contour lines), $\theta=\pi k / 16(k=0,1, \ldots, 31)$, $\rho=(0.2,0.4,0.9)$, and $n=100$.

correlation coefficients $\rho$, levels $\alpha$, and domain points $x$. In fact, the asymptotics of Corollary 2.2 appear to take effect already at sample sizes of 100 , irrespective of the values of $u, \rho, \alpha$, and $x$.

To get an impression of how the $u$-th geometric conditional quantiles behave over a range of different values of $u$, we simulated 100 observations from the trivariate normal distribution $N(0, \Sigma)$ with $\rho=(0.2,0.4,0.9)$. We take $u=r(\cos \theta, \sin \theta)^{T}$, with $r=0.1,0.2, \ldots, 0.9$ and $\theta=\pi k / 16(k=0,1, \ldots, 31)$. For each $r$, we computed $\hat{Q}_{n}(u \mid x)$ corresponding to the index vector $u$ at the domain point $x=0$. Figure 2 shows the result in each case. Note that the enclosed area is increasing with increasing values of $r$. Thus the surfaces are neatly nested. It indicates that $\hat{Q}_{n}(u \mid x)$ is equivariant under linear reparametrization of the design. Further we see that conditional quantiles are not lying on concentric circles, as this should be the case if the 
data points are i.i.d. Thus, the extent each surface deviates from a circle provides a useful way for assessing departures from model assumptions like i.i.d. and "heteroskedasticity". In general, the regions enclosed by conditional quantile contours can be viewed as multivariate analogues of QQ-plots used for univariate unconditional data analysis. They are useful for detecting outliers in multivariate data.

\section{Application}

In this section we compute geometric conditional quantiles using a data set consisting of 19 variables on 403 subjects from 1046 subjects who were interviewed in a study to understand the prevalence of obesity, diabetes, and other cardiovascular risk factors in central Virginia for African-Americans; see http://hesweb1.med.virginia.edu/biostat/s/data/. It was observed that Diabetes Mellitus Type II (adult onset diabetes) is associated most strongly with obesity. From the set of variables we consider two responses: systolic (higher) blood pressure (BPS), and diastolic (lower) blood pressure (BPD). Both variables are measured in $m m \mathrm{Hg}$. Averages of BPS were taken if more than one measurement was available. As one would expect, the correlation between BPS and BPD is high (0.608). It is well-known to physiologists that systolic and diastolic blood pressure jointly vary with Age and Weight. Therefore, as a first covariate we consider Age with a range from 19 to 92 years. As a second covariate we calculated from reported heights and weights the body mass index (BMI) in $\mathrm{kg} / \mathrm{m}^{2}$. These values ranged from 16.04 to 55.9. Observations that had missing values in any of the 5 variables were omitted from further analysis, leaving 378 out of the 403 original observations available.

According to the guidelines from the second US National Health and Nutrition Examination Survey (NHANES II), a man with a BMI between 27.8 and $31.1 \mathrm{~kg} / \mathrm{m}^{2}$ and a women with a BMI between 27.3 and $32.3 \mathrm{~kg} / \mathrm{m}^{2}$ can be considered to have overweight. Combining these limits and taking averages, we noted that $84(22.2 \%)$ respondents in the analysed data set have overweight whereas $115(30.4 \%)$ respondents are severely overweighed with a BMI $>31.7 \mathrm{~kg} / \mathrm{m}^{2}$. Given this information, the domain points for BMI were set at 20, 25, 30, 35, 40, and $45 \mathrm{~kg} / \mathrm{m}^{2}$, respectively.

Table 1 shows estimated $u$-th geometric conditional quantiles for some selected values of $u$. If the values of the coordinates of the index vector $u$ are both positive, it indicates that both BPS and BPD are higher than "normal". Similarly, if the values of the coordinates of $u$ are both negative, it indicates a direction toward which both BPS and BPD are lower than normal. 
Table 1: $u$-th geometric conditional quantile estimates for blood pressure data given values of the covariates Age and BMI; asymptotic standard errors are in parentheses; $u=(0.0,0.0)^{T}$ represents the geometric conditional median.

\begin{tabular}{|c|c|c|c|c|c|c|c|}
\hline \multirow[t]{2}{*}{ Covariate } & \multirow[t]{2}{*}{$x$} & \multicolumn{2}{|c|}{$u=(0.0,0.0)^{T}$} & \multicolumn{2}{|c|}{$u=(0.8,0.4)^{T}$} & \multicolumn{2}{|c|}{$u=(-0.8,-0.4)^{T}$} \\
\hline & & BPS & $\mathrm{BPD}$ & BPS & $\mathrm{BPD}$ & BPS & $\mathrm{BPD}$ \\
\hline \multirow[t]{6}{*}{ Age } & 30 & $\begin{array}{c}127.230 \\
(0.125)\end{array}$ & $\begin{array}{r}81.099 \\
(0.326)\end{array}$ & $\begin{array}{c}148.605 \\
(1.862)\end{array}$ & $\begin{array}{c}88.752 \\
(1.569)\end{array}$ & $\begin{array}{r}107.561 \\
(0.177)\end{array}$ & $\begin{array}{l}75.195 \\
(0.292)\end{array}$ \\
\hline & 40 & $\begin{array}{c}132.555 \\
(0.196)\end{array}$ & $\begin{array}{r}84.796 \\
(0.299)\end{array}$ & $\begin{array}{c}158.868 \\
(1.128)\end{array}$ & $\begin{array}{c}91.832 \\
(0.345)\end{array}$ & $\begin{array}{c}109.242 \\
(0.129)\end{array}$ & $\begin{array}{c}77.008 \\
(0.156)\end{array}$ \\
\hline & 50 & $\begin{array}{c}138.052 \\
(0.083)\end{array}$ & $\begin{array}{l}86.728 \\
(0.264)\end{array}$ & $\begin{array}{c}171.185 \\
(1.381)\end{array}$ & $\begin{array}{c}93.744 \\
(0.342)\end{array}$ & $\begin{array}{r}113.207 \\
(0.716)\end{array}$ & $\begin{array}{r}78.136 \\
(0.340)\end{array}$ \\
\hline & 60 & $\begin{array}{c}142.235 \\
(0.197)\end{array}$ & $\begin{array}{r}82.798 \\
(0.157)\end{array}$ & $\begin{array}{r}177.887 \\
(1.434)\end{array}$ & $\begin{array}{c}91.086 \\
(0.395)\end{array}$ & $\begin{array}{c}118.485 \\
(0.478)\end{array}$ & $\begin{array}{l}76.421 \\
(0.139)\end{array}$ \\
\hline & 70 & $\begin{array}{r}145.708 \\
(0.158)\end{array}$ & $\begin{array}{r}81.665 \\
(0.242)\end{array}$ & $\begin{array}{r}178.917 \\
(4.165)\end{array}$ & $\begin{array}{r}89.241 \\
(0.327)\end{array}$ & $\begin{array}{r}119.600 \\
(0.612)\end{array}$ & $\begin{array}{r}75.151 \\
(0.451)\end{array}$ \\
\hline & 80 & $\begin{array}{c}150.131 \\
(1.054)\end{array}$ & $\begin{array}{l}82.133 \\
(1.644)\end{array}$ & $\begin{array}{r}178.961 \\
(0.623)\end{array}$ & $\begin{array}{c}88.390 \\
(0.250)\end{array}$ & $\begin{array}{c}123.554 \\
(0.979)\end{array}$ & $\begin{array}{c}76.452 \\
(0.374)\end{array}$ \\
\hline \multirow[t]{6}{*}{ BMI } & 20 & $\begin{array}{c}133.855 \\
(0.298)\end{array}$ & $\begin{array}{l}81.579 \\
(0.179)\end{array}$ & $\begin{array}{c}168.858 \\
(7.167)\end{array}$ & $\begin{array}{c}89.166 \\
(3.314)\end{array}$ & $\begin{array}{r}110.305 \\
(0.221)\end{array}$ & $\begin{array}{r}75.035 \\
(0.227)\end{array}$ \\
\hline & 25 & $\begin{array}{r}135.052 \\
(0.218)\end{array}$ & $\begin{array}{l}82.518 \\
(0.223)\end{array}$ & $\begin{array}{r}169.276 \\
(2.266)\end{array}$ & $\begin{array}{c}89.725 \\
(0.524)\end{array}$ & $\begin{array}{r}110.217 \\
(0.222)\end{array}$ & $\begin{array}{r}75.320 \\
(0.170)\end{array}$ \\
\hline & 30 & $\begin{array}{r}136.209 \\
(0.152)\end{array}$ & $\begin{array}{r}83.631 \\
(0.267)\end{array}$ & $\begin{array}{c}169.775 \\
(1.230)\end{array}$ & $\begin{array}{c}90.496 \\
(0.248)\end{array}$ & $\begin{array}{r}110.571 \\
(0.518)\end{array}$ & $\begin{array}{l}75.939 \\
(0.315)\end{array}$ \\
\hline & 35 & $\begin{array}{r}137.027 \\
(0.372)\end{array}$ & $\begin{array}{l}84.740 \\
(0.347)\end{array}$ & $\begin{array}{c}169.784 \\
(2.401)\end{array}$ & $\begin{array}{c}91.272 \\
(1.515)\end{array}$ & $\begin{array}{r}110.740 \\
(0.670)\end{array}$ & $\begin{array}{c}76.619 \\
(0.216)\end{array}$ \\
\hline & 40 & $\begin{array}{r}137.667 \\
(0.251)\end{array}$ & $\begin{array}{r}85.631 \\
(0.190)\end{array}$ & $\begin{array}{c}169.826 \\
(8.515)\end{array}$ & $\begin{array}{c}91.928 \\
(3.701)\end{array}$ & $\begin{array}{c}110.799 \\
(0.167)\end{array}$ & $\begin{array}{c}77.588 \\
(0.201)\end{array}$ \\
\hline & 45 & $\begin{array}{r}139.062 \\
(1.569)\end{array}$ & $\begin{array}{r}86.467 \\
(0.494)\end{array}$ & $\begin{array}{l}169.931 \\
(37.877)\end{array}$ & $\begin{array}{r}92.327 \\
(12.609)\end{array}$ & $\begin{array}{r}110.719 \\
(0.528)\end{array}$ & $\begin{array}{r}78.551 \\
(0.272)\end{array}$ \\
\hline
\end{tabular}

Observe that the $u$-th geometric conditional quantile estimates, of BPS and BPD increase with increasing values of Age. The associated asymptotic conditional standard errors, obtained from Corollary 2.2, seem to confirm this. On the other hand, it is interesting to see that the $u$-th geometric conditional quantiles of BPS and BPD increase slowly with increasing values of BMI. Only for large values of BMI, say 40 and $45 \mathrm{~kg} / \mathrm{m}^{2}$, the conditional standard errors become large.

Figures 3.a)-3.d) show scatter-plots of BPS and BPD versus both Age and BMI. Plotted in each figure is the conditional median curve (median-dashed lines), represented by the case $u=(0.0,0.0)^{T}$ in Table 1 . We see that nearly a linear relationship between the variables. Also plotted in Figures 3.a)-3.d) are the $u=(0.8,0.58)^{T}(\|u\|=0.9764)$ conditional quantiles 
a)

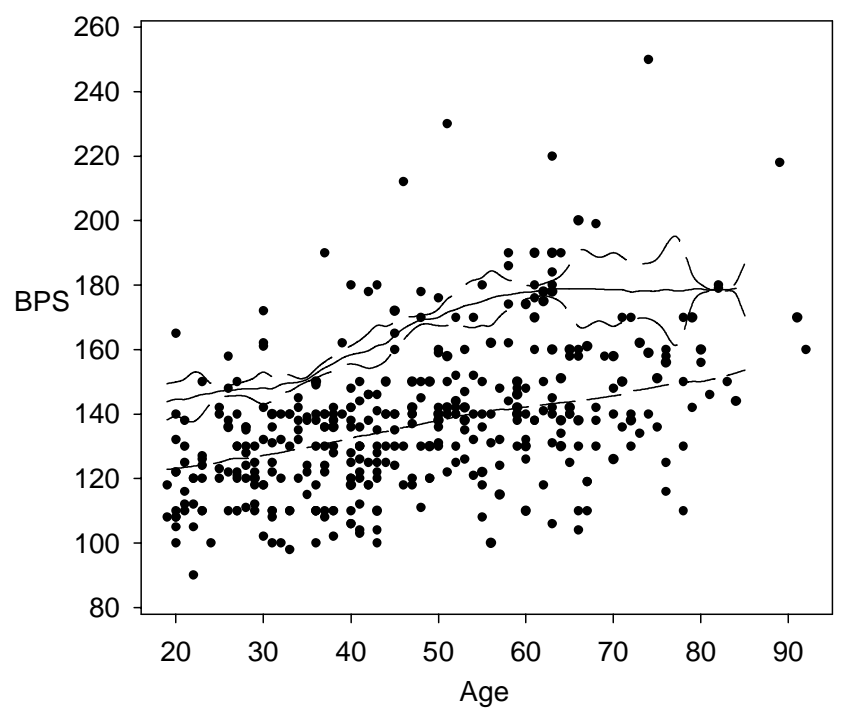

c)

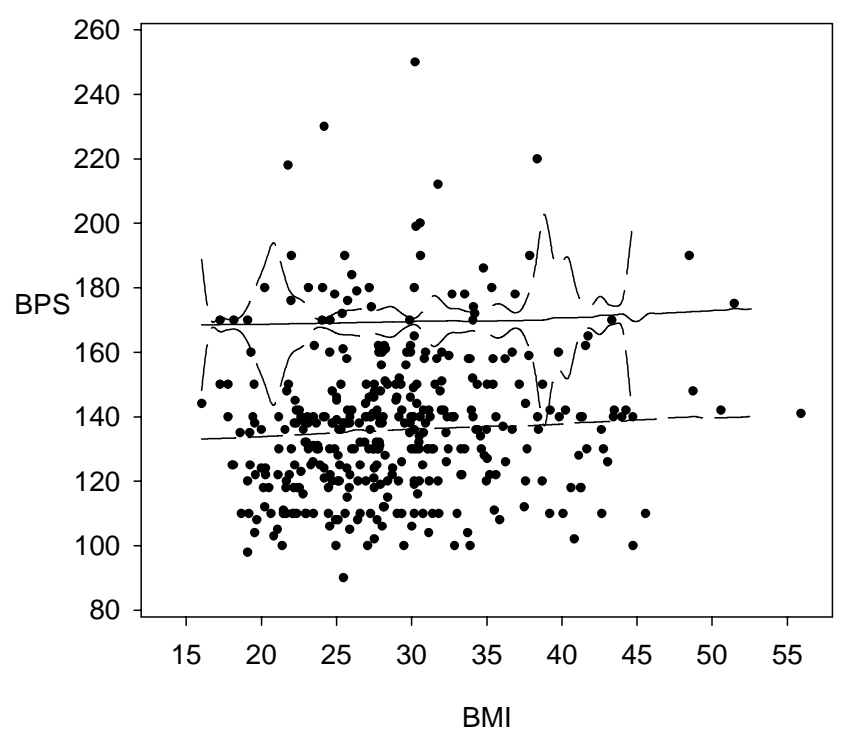

b)

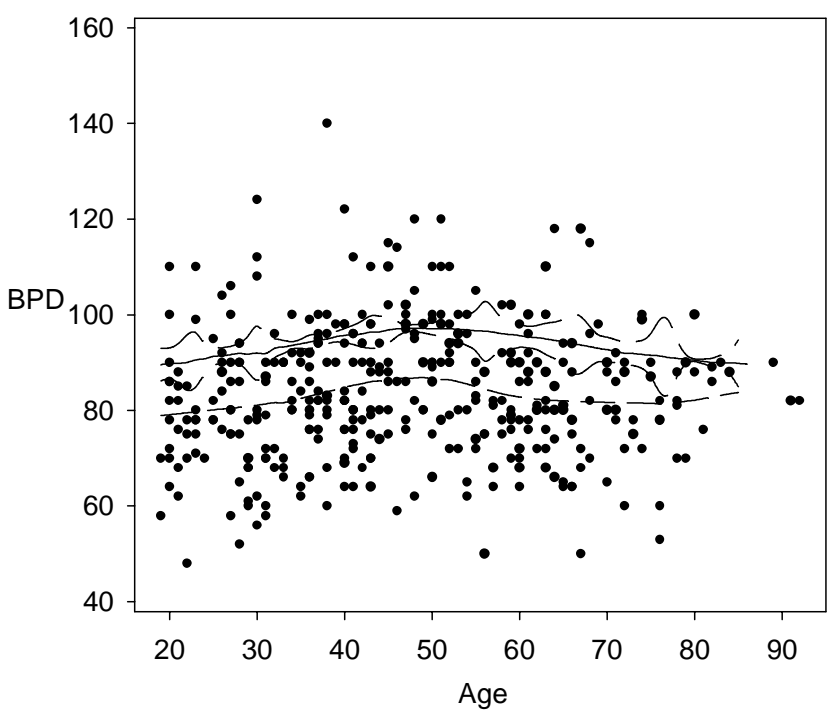

d)

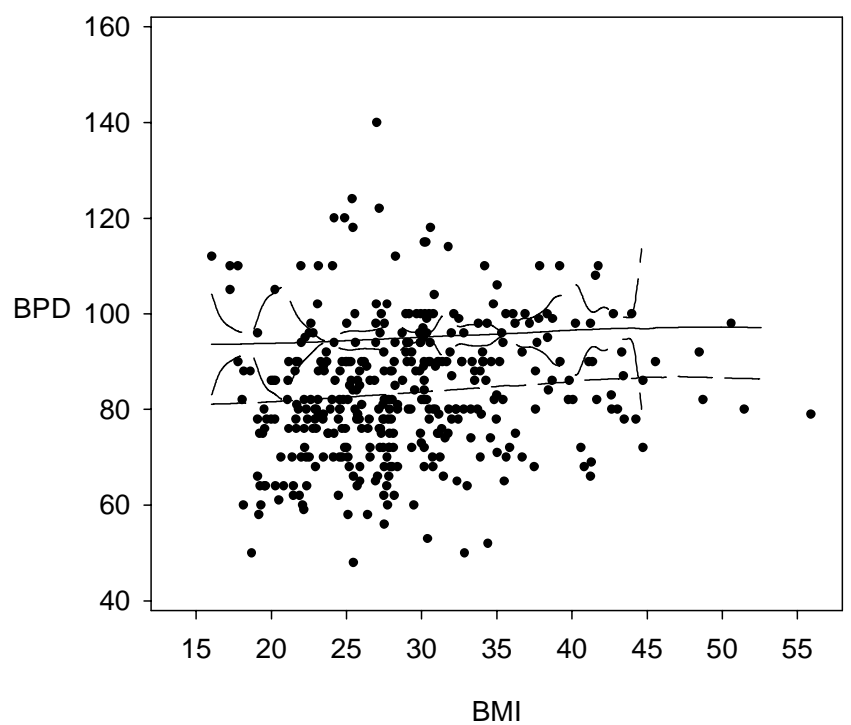

Figure 3: Scatter-plots of BPS and BPD versus Age and BMI. Median-dashed lines are conditional median curves; solid lines are $\hat{Q}_{n}(u \mid x)$ 's with $u=(0.8,0.58)^{T}$ and long-dashed lines are the associated asymptotic 95\% confidence bands. 
(solid lines) surrounded by the associated 95\% conditional confidence bands (long-dashed lines) obtained from Corollary 2.2.

With $\|u\|=0.9764$, we observe from Figure 3.a) that BPS ranges between 143 and 179 $\mathrm{mm} \mathrm{Hg}$ in a nonlinear fashion. Similarly, from Figure 3.b), we see that BPD ranges between 89.2 and $97.2 \mathrm{~mm} \mathrm{Hg}$. Using BMI as a covariate, Figures 3.c)-3.d) show values of BPS in the range [168.6, 173.8] $\mathrm{mm} \mathrm{Hg}$ and $\mathrm{BPD}$ in the range [93.6, 97.2] $\mathrm{mm} \mathrm{Hg}$. In NHANES II the criterion for determining a respondent with a definite too high blood pressure was fixed at an average value of $160 \mathrm{~mm} \mathrm{Hg}$ for BPS and $95 \mathrm{~mm} \mathrm{Hg}$ for BPD. Clearly, these values are based on blood pressure data sets observed in the past covering all possible values of BMI. The results, given in Table 1 in columns 5-8, suggest that a much "finer" way of classifying a respondent can be based on the geometric conditional quantile and the associated conditional standard deviation, with the conditioning on individual BMI values rather than using all BMI values jointly in an unconditional way.

\section{Summary}

We have established a Bahadur type linear representation of the geometric conditional quantile estimator. From this result the asymptotic normality for the estimator was derived. We have demonstrated by simulation that in the case $d=2$ and $s=1$ the resulting confidence ellipses for the geometric conditional quantile are adequate in finite sample situations, even in the case of extreme quantiles. Finally, we showed that the geometric conditional quantile and its associated conditional standard deviation can be used to "fine-tune" classification of respondents having a too high blood pressure.

\section{Appendix A: Proofs of the Main Results}

\section{A.1 Some lemmas}

We first state a basic inequality, which can be viewed as an extension of Fact 5.1 of Chaudhuri (1992) to the multivariate case. It plays an important role in proving the main results.

Fact A.1. Let $Z_{1}, Z_{2}, \ldots, Z_{n}$ be a sequence of $d$-dimensional i.i.d. random vectors and $p\left(y_{1}, y_{2}, \ldots, y_{m}\right)$ be a symmetric d-dimensional kernel such that $\|p(\cdot)\| \leq b$ for a positive constant $b$. Assume that $E\left[p\left(Z_{1}, Z_{2}, \ldots, Z_{m}\right)\right]=0$ and $\operatorname{Var}\left(p\left(Z_{1}, Z_{2}, \ldots, Z_{m}\right)\right)=\left(\sigma_{i j}\right)_{d \times d}$. 
Define the $U$-statistic as

$$
U_{n}=\frac{1}{C_{n}^{m}} \sum_{1 \leq i_{1}, i_{2}, \ldots, i_{m} \leq n} p\left(Z_{i_{1}}, Z_{i_{2}}, \ldots, Z_{i_{m}}\right) .
$$

Then, for each $t>0$, it holds that

$$
\mathbb{P}\left\{\left\|U_{n}\right\|>t\right\} \leq 2 d \exp \left\{-\frac{\left[\frac{n}{m}\right] t^{2}}{2 d^{2} \max _{1 \leq l \leq d} \sigma_{l l}+2 d b t / 3}\right\} .
$$

Proof. Let $e_{l}(l=1,2, \ldots, d)$ be the $d$-dimensional unit vector in which the $l$-th component is equal to 1. According to Fact 5.1 of Chaudhuri (1992), it holds that

$$
\mathbb{P}\left\{\left\|U_{n}\right\|>t\right\} \leq \sum_{l=1}^{d} \mathbb{P}\left\{\left|e_{l}^{T} U_{n}\right|>\frac{t}{d}\right\} \leq 2 \sum_{l=1}^{d} \exp \left\{-\frac{\left[\frac{n}{m}\right]\left(\frac{t}{d}\right)^{2}}{2 \cdot \sigma_{l l}+2 b \cdot \frac{t}{d} / 3}\right\},
$$

which results in Fact A.1.

The proof of Theorem 2.1 is based on the following sequence of lemmas. These lemmas themselves are also of interest to characterize the properties of the estimated geometric conditional quantile $\hat{Q}_{n}(u \mid x)$. We first show that $\hat{Q}_{n}(u \mid x)$ is bounded asymptotically by some constant with probability one.

Lemma A.2. Under Condition 4, $\ddot{a})$ and $\dddot{x})$ of Condition 2 and Condition 3, there exists a constant $K_{1}=K_{1}(u)>0$ such that

$$
\left\|\hat{Q}_{n}(u \mid x)-Q(u \mid x)\right\| \leq K_{1}
$$

holds almost surely for all sufficiently large $n$.

Proof. First, we can choose some suitable constant $K_{1}^{*}>0$ such that

$$
\mathbb{P}\left(\|Y\|>\frac{K_{1}^{*}}{4} \mid X=x\right) \leq \frac{1-\|u\|}{3+\|u\|}
$$

From Theorem 4.2 of Devroye (1981), if we view the regression function there as the conditional probability $P\left(\|Y\|>\frac{K_{1}^{*}}{4} \mid X=x\right)$, then the following asymptotic relationship

$$
\sum_{i=1}^{n} w_{n, i} I_{\left(\left\|Y_{i}\right\|>\frac{K_{1}^{*}}{4}\right)} \rightarrow P\left(\|Y\|>\frac{K_{1}^{*}}{4} \mid X=x\right)
$$

holds with probability one. It is easy to see that

$$
\begin{aligned}
& \mid \sum_{i=1}^{n}\left[\Phi\left(u, Y_{i}+\theta-Q(u \mid x)\right)-\Phi\left(u, Y_{i}-Q(u \mid x)\right)\right] K\left(\frac{x-X_{i}}{h_{n}}\right) \mathbb{I}{\left(\left\|Y_{i}-Q(u \mid x)\right\|>\frac{K_{1}^{*}}{4}\right) \mid}_{\leq}(1+\|u\|)\|\theta\| \sum_{i=1}^{n} K\left(\frac{x-X_{i}}{h_{n}}\right) \mathbb{I}{ }_{\left(\left\|Y_{i}-Q(u \mid x)\right\|>\frac{K_{1}^{*}}{4}\right)}
\end{aligned}
$$


If $\Phi(u, \theta)>K_{1}^{*}$, then it holds that

$$
\begin{aligned}
& \sum_{i=1}^{n}\left[\Phi\left(u, Y_{i}+\theta-Q(u \mid x)\right)-\Phi\left(u, Y_{i}-Q(u \mid x)\right)\right] K\left(\frac{x-X_{i}}{h_{n}}\right) \mathbb{I}{\left(\left\|Y_{i}-Q(u \mid x)\right\| \leq \frac{K_{1}^{*}}{4}\right)}_{>} \\
> & \frac{\Phi(u, \theta)}{2} \sum_{i=1}^{n} K\left(\frac{x-X_{i}}{h_{n}}\right) \mathbb{I}{ }_{\left(\left\|Y_{i}-Q(u \mid x)\right\| \leq \frac{K_{1}^{*}}{4}\right)} \\
\geq & \frac{(1-\|u\|)\|\theta\|}{2} \sum_{i=1}^{n} K\left(\frac{x-X_{i}}{h_{n}}\right) \mathbb{I}{ }_{\left(\left\|Y_{i}-Q(u \mid x)\right\| \leq \frac{K_{1}^{*}}{4}\right)}
\end{aligned}
$$

From (A.3) and (A.4) above, we know that, if $\Phi(u, \theta)>K_{1}^{*}$, it holds that

$$
\begin{aligned}
& \sum_{i=1}^{n}\left(\Phi\left(u, Y_{i}+\theta-Q(u \mid x)\right)-\Phi\left(u, Y_{i}-Q(u \mid x)\right)\right) K\left(\frac{x-X_{i}}{h_{n}}\right) \\
> & -(1+\|u\|)\|\theta\| \sum_{i=1}^{n} K\left(\frac{x-X_{i}}{h_{n}}\right) \mathbb{I}\left(\left\|Y_{i}-Q(u \mid x)\right\|>\frac{K_{1}^{*}}{4}\right) \\
& +\frac{(1-\|u\|)\|\theta\|}{2} \sum_{i=1}^{n} K\left(\frac{x-X_{i}}{h_{n}}\right) \mathbb{I}\left(\left\|Y_{i}-Q(u \mid x)\right\| \leq \frac{K_{1}^{*}}{4}\right) \\
= & \frac{(3+\|u\|)\|\theta\|}{2} \sum_{i=1}^{n} K\left(\frac{x-X_{i}}{h_{n}}\right)\left(\frac{1-\|u\|}{3+\|u\|}-\sum_{i=1}^{n} w_{n, i} \mathbb{I}\left(\left\|Y_{i}-Q(u \mid x)\right\|>\frac{K_{1}^{*}}{4}\right)\right) .
\end{aligned}
$$

In view of (A.1) and (A.2), it can be shown that (A.5) is excess zero for all sufficiently large $n$. Hence, from the definition of $\hat{Q}_{n}(u \mid x)$, it holds that

$$
\Phi\left(u, \hat{Q}_{n}(u \mid x)-Q(u \mid x)\right) \leq K_{1}^{*}
$$

Noting that $\|u\|<1$, we obtain that Lemma A.2 holds.

Further, we aim to prove that the $u$-th geometric conditional quantile $\hat{Q}_{n}(u \mid x)$ converges at a special rate stated in Lemma A.7 below. In doing so, we now show the following four lemmas. In the sequel, for simplicity of presentation, let $Q(u \mid x)=\left(q_{1}, \ldots, q_{d}\right)^{T}$ and $\left[n^{\alpha}\right]$ is the greatest integer less than $n^{\alpha}$ for some constant $\alpha>0$. Also, $C>0$ denotes a constant which may take different values in different places.

Lemma A.3. Assume that Conditions 2 to 4 and (2.1) hold. For some constant $\alpha>0$, let $B_{n}$ be the subset of $\mathbb{R}^{d}$ defined as

$$
\begin{aligned}
B_{n}= & \left\{\left(v_{1}-q_{1}, \ldots, v_{d}-q_{d}\right)^{T} \mid\left[n^{\alpha}\right]\left(v_{i}-q_{i}\right)=\right.\text { an integer } \\
& \text { and } \left.\left|v_{i}-q_{i}\right| \leq K_{1} \text { for all } 1 \leq i \leq d\right\},
\end{aligned}
$$


then there exists a constant $K_{2}>0$ such that

$$
\begin{aligned}
& \max _{\theta \in B_{n}} \frac{1}{n \mathbb{E} K\left(\frac{x-X}{h_{n}}\right)} \| \sum_{i=1}^{n}\left[K\left(\frac{x-X_{i}}{h_{n}}\right) U\left(Y_{i}-\theta-Q(u \mid x)\right)\right. \\
& \left.-\mathbb{E} K\left(\frac{x-X_{i}}{h_{n}}\right) U\left(Y_{i}-\theta-Q(u \mid x)\right)\right] \| \leq K_{2}\left(\frac{\log n}{n h_{n}^{s}}\right)^{\frac{1}{2}}
\end{aligned}
$$

holds almost surely for all sufficiently large $n$.

Proof. From the definition of $B_{n}$, for all sufficiently large $n$, the number of the elements in it is less than $\gamma_{1} n^{\alpha d}$ for some constant $\gamma_{1}$ which is related to the constant $K_{1}$. It can be shown directly that

$$
\mathbb{E} K\left(\frac{x-X}{h_{n}}\right) \sim h_{n}^{s} g(x) \text { and } \mathbb{E} K^{2}\left(\frac{x-X}{h_{n}}\right) \sim h_{n}^{s} g(x) \int K^{2}(t) d t .
$$

Let $E_{1 n}$ be the event defined in (A.6). According to Fact A.1 and (A.7), for some constant $C=C(x)$, it holds that

$$
\begin{aligned}
\mathbb{P}\left(E_{1 n}^{c}\right) & \leq \gamma_{1} n^{\alpha d} \cdot 2 d \exp \left\{-\frac{n\left(\mathbb{E} K\left(\frac{x-X}{h_{n}}\right) K_{2} \sqrt{\frac{\log n}{n h_{n}^{s}}}\right)^{2}}{2 d^{2} \mathbb{E} K^{2}\left(\frac{x-X}{h_{n}}\right)+\frac{2}{3} d \cdot 2 c_{2} \cdot \mathbb{E} K\left(\frac{x-X}{h_{n}}\right) K_{2} \sqrt{\frac{\log n}{n h_{n}^{s}}}}\right\} \\
& \leq 2 d \gamma_{1} \exp \left\{\left(\alpha d-C K_{2}^{2}\right) \log n\right\} .
\end{aligned}
$$

Through choosing a suitable constant $K_{2}=K_{2}(x)$, we can obtain $\sum_{n=1}^{\infty} \mathbb{P}\left(E_{n}^{c}\right)<\infty$. Accordingly, by the Borel-Cantelli lemma, (A.6) holds.

Lemma A.4. It holds almost surely that

$$
\left\|\sum_{i=1}^{n} w_{n, i}\left(U\left(Y_{i}-\hat{Q}_{n}(u \mid x)\right)+u\right)\right\| \leq \max _{1 \leq i \leq n} w_{n, i} .
$$

Proof. Analogous to the proof of Theorem 2.1.2 of Chaudhuri (1996), for any $h \in \mathbb{R}^{d}$, by the definition of $\hat{Q}_{n}(u \mid x)$, it holds that

$$
\begin{array}{r}
\sum_{1 \leq i \leq n, Y_{i} \neq \hat{Q}_{n}(u \mid x)} w_{n, i}\left\{\left\langle U\left(Y_{i}-\hat{Q}_{n}(u \mid x)\right), h\right\rangle+\langle u, h\rangle\right\} \\
+\sum_{1 \leq i \leq n, Y_{i}=\hat{Q}_{n}(u \mid x)} w_{n, i}\{\|h\|+\langle u, h\rangle\} \geq 0 .
\end{array}
$$

Because $\left(X_{i}, Y_{i}\right)(i=1,2, \ldots, n)$ are absolute continuous random variables, $Y_{i}(i=1,2, \ldots, n)$ do not equal to each other almost surely. Then, by the property that $h$ is arbitrary in $\mathbb{R}^{d},(\mathrm{~A} .8)$ holds. 
Lemma A.5. Under Conditions 2 to 4 , there exists constant $K_{3}>0$ such that

$$
\max _{\theta \in B_{n}}\left|\frac{1}{n \mathbb{E} K\left(\frac{x-X}{h_{n}}\right)} \sum_{i=1}^{n} K\left(\frac{x-X_{i}}{h_{n}}\right) \mathbb{I}_{\left(\left\|Y_{i}-\theta-Q(u \mid x)\right\| \leq n^{-\beta}\right)}\right| \leq K_{3} \frac{\log n}{n h_{n}^{s}}
$$

holds almost surely for all sufficiently large $n$ and $\beta>\frac{\gamma}{d}$.

Proof. For any $\theta$ in $B_{n}$, noting the bound of the supported set of $K(\cdot)$ and i) of Condition 2, we obtain that

$$
\begin{aligned}
& \mathbb{E} K\left(\frac{x-X}{h_{n}}\right) \mathbb{I}_{\left(\|Y-\theta-Q(u \mid x)\| \leq n^{-\beta}\right)} \\
= & h_{n}^{s} \iint K(z) \mathbb{I}_{\left(\|y-\theta-Q(u \mid x)\| \leq n^{-\beta}\right)} f\left(x-h_{n} z, y\right) d z d y \\
\leq & C h_{n}^{s} \int \mathbb{I}_{\left(\|y-\theta-Q(u \mid x)\| \leq n^{-\beta}\right)} d y \leq C n^{-d \beta} h_{n}^{s} .
\end{aligned}
$$

From (A.10), (A.7) and $\beta>\frac{\gamma}{d}$, it is easy to see that

$$
\mathbb{E} K\left(\frac{x-X}{h_{n}}\right) \mathbb{I}_{\left(\|Y-\theta-Q(u \mid x)\| \leq n^{-\beta}\right)}=o\left(\mathbb{E} K\left(\frac{x-X}{h_{n}}\right) \frac{\log n}{n h_{n}^{s}}\right) .
$$

For any $\theta$ in $B_{n}$, analogous to (A.10), it holds that

$$
\operatorname{var}\left(K\left(\frac{x-X_{i}}{h_{n}}\right) \mathbb{I}_{\left(\left\|Y_{i}-\theta-Q(u \mid x)\right\| \leq n^{-\beta}\right)}\right) \leq C n^{-d \beta} h_{n}^{s} .
$$

Denote the event (A.9) by $E_{2 n}$. From (A.7), (A.11), (A.12) and Bernstein's inequality (see Serfling, 1980), we get that

$$
\begin{aligned}
& \mathbb{P}\left(E_{2 n}^{c}\right) \\
\leq & 2 \gamma_{1} n^{\alpha d} \exp \left\{\frac{-\left(n \mathbb{E} K\left(\frac{x-X}{h_{n}}\right) K_{3} \frac{\log n}{n h_{n}^{s}}\right)^{2}}{2 n v a r\left(K\left(\frac{x-X_{i}}{h_{n}}\right) \mathbb{I}_{\left(\left\|Y_{i}-\theta-Q(u \mid x)\right\| \leq n^{-\beta}\right)}\right)+\frac{2}{3} \cdot 2 c_{2} \cdot n \mathbb{E} K\left(\frac{x-X}{h_{n}}\right) K_{3} \frac{\log n}{n h_{n}^{s}}}\right\} \\
\leq & 2 \gamma_{1} \exp \left\{\left(\alpha d-C K_{3}\right) \log n\right\} .
\end{aligned}
$$

Analogous to the proof of Lemma A.3, (A.9) holds.

The following Lemma A.6, which may be known somewhere, can be inferred directly from Bernstein's inequality, Borel-Cantelli lemma and (A.7).

Lemma A.6. Under Conditions 3, 4 and ii) of Condition 2, it holds that

$$
\frac{\sum_{i=1}^{n} K\left(\frac{x-X_{i}}{h_{n}}\right)}{n \mathbb{E} K\left(\frac{x-X}{h_{n}}\right)}-1=O\left(\sqrt{\frac{\log n}{n h_{n}^{s}}}\right) \quad \text { a.s. }
$$


From the lemmas above, we can argue the convergence rate of the estimated $u$-th geometric conditional quantile $\hat{Q}_{n}(u \mid x)$. In the rest of this subsection, the following constants $\alpha$ and $\beta$ are restricted to

$$
\left(1+\frac{1}{d}\right) \gamma<\beta+\gamma<\alpha<\frac{(1-\gamma) \min \left(\lambda_{1}, \lambda_{2}\right)}{s}
$$

Lemma A.7. Under Conditions 2 to 4 and (2.1), there exists a constant $K_{4}>0$ such that almost surely $\left\|\hat{Q}_{n}(u \mid x)-Q(u \mid x)\right\| \leq K_{4}\left(\log n /\left(n h_{n}^{s}\right)\right)^{1 / 2}$ for all $n$ sufficiently large.

Proof. Because of the bound of $K(\cdot)$, it can be shown directly that

$$
\max _{1 \leq i \leq n} K\left(\frac{x-X_{i}}{h_{n}}\right)=o(\sqrt{\log n}) .
$$

Assume that $\theta_{n}^{*}$ is the nearest point to $\hat{Q}_{n}(u \mid x)$ in $B_{n}$. Going along the same lines as in Chaudhuri (1992), in the case of $\left\|Y_{i}-\theta_{n}^{*}-Q(u \mid x)\right\|>n^{-\beta}$, because

$$
\left\|\hat{Q}_{n}(u \mid x)-\theta_{n}^{*}-Q(u \mid x)\right\| \leq \gamma_{3} n^{-\alpha}
$$

for some constant $\gamma_{3}>0$, it holds that

$$
\begin{aligned}
& \left\|U\left(Y_{i}-\hat{Q}_{n}(u \mid x)\right)-U\left(Y_{i}-\theta_{n}^{*}-Q(u \mid x)\right)\right\| \\
\leq & 2\left\|\hat{Q}_{n}(u \mid x)-\theta_{n}^{*}-Q(u \mid x)\right\| \cdot\left\|Y_{i}-\theta_{n}^{*}-Q(u \mid x)\right\|^{-1} \leq 2 \gamma_{3} n^{-(\alpha-\beta)} .
\end{aligned}
$$

From the two relationships above, Lemma A.6, (A.13), (A.9) and (A.8), for all sufficiently large $n>0$, the following result can be derived almost surely

$$
\begin{aligned}
& \left\|\frac{1}{n \mathbb{E} K\left(\frac{x-X}{h_{n}}\right)} \sum_{i=1}^{n} K\left(\frac{x-X_{i}}{h_{n}}\right)\left(U\left(Y_{i}-\theta_{n}^{*}-Q(u \mid x)\right)+u\right)\right\| \\
\leq & \left\|\frac{1}{n \mathbb{E} K\left(\frac{x-X}{h_{n}}\right)} \sum_{i=1}^{n} K\left(\frac{x-X_{i}}{h_{n}}\right)\left[U\left(Y_{i}-\theta_{n}^{*}-Q(u \mid x)\right)-U\left(Y_{i}-\hat{Q}_{n}(u \mid x)\right)\right]\right\| \\
& +\frac{1}{n \mathbb{E} K\left(\frac{x-X}{h_{n}}\right)}\left\|\sum_{i=1}^{n} K\left(\frac{x-X_{i}}{h_{n}}\right)\left(U\left(Y_{i}-\hat{Q}_{n}(u \mid x)\right)+u\right)\right\| \\
\leq & \frac{2}{n \mathbb{E} K\left(\frac{x-X}{h_{n}}\right)} \sum_{i=1}^{n} K\left(\frac{x-X_{i}}{h_{n}}\right) \mathbb{I}_{\left(\left\|Y_{i}-\theta_{n}^{*}-Q(u \mid x)\right\| \leq n^{-\beta}\right)} \\
& +\frac{1}{n \mathbb{E} K\left(\frac{x-X}{h_{n}}\right)} \sum_{i=1}^{n} K\left(\frac{x-X_{i}}{h_{n}}\right) \cdot 2 \gamma_{3} n^{-(\alpha-\beta)}+\frac{1}{n \mathbb{E} K\left(\frac{x-X}{h_{n}}\right)} \max _{1 \leq i \leq n} K\left(\frac{x-X_{i}}{h_{n}}\right) \\
\leq & C \frac{\log n}{n h_{n}^{s}} .
\end{aligned}
$$


We now begin to prove that the following asymptotic relationship

$$
\frac{\left\|\mathbb{E} K\left(\frac{x-X}{h_{n}}\right)(U(Y-\theta-Q(u \mid x))+u)\right\|}{\mathbb{E} K\left(\frac{x-X}{h_{n}}\right)} \sim\|\mathbb{E}[U(Y-\theta-Q(u \mid x))+u \mid X=x]\|
$$

holds uniformly for $\theta \in B_{n}$. For any $\theta_{n} \in B_{n}$, through the variable substitution, Condition 3 and $(2.1)$, we can see that

$$
\begin{aligned}
& \| \mathbb{E} K\left(\frac{x-X}{h_{n}}\right)\left(U\left(Y-\theta_{n}-Q(u \mid x)\right)+u\right) \\
& -h_{n}^{s} g(x) \mathbb{E}\left[U\left(Y-\theta_{n}-Q(u \mid x)\right)+u \mid X=x\right] \| \\
\leq & h_{n}^{s}\left\|\iint K(z)\left[U\left(y-\theta_{n}-Q(u \mid x)\right)+u\right]\left[f\left(x-h_{n} z, y\right)-f(x, y)\right] d z d y\right\| \\
\leq & 2 h_{n}^{s} \int K(z)\left(\int\left|f\left(x-h_{n} z, y\right)-f(x, y)\right| d y\right) d z=O\left(h_{n}^{s+\lambda_{1}}\right) .
\end{aligned}
$$

Similar to the proof of Lemma 5.3 of Chaudhuri (1992), from the definition of the $u$-th geometric conditional quantile $Q(u \mid x)$, it is not difficult to show that

$$
\mathbb{E}[U(Y-Q(u \mid x))+u \mid X=x]=0 .
$$

Then, by Taylor's expansion and the equation above, it holds that

$$
\begin{aligned}
& \frac{h_{n}^{\lambda_{1}}}{\left\|\int\left(U\left(y-\theta_{n}-Q(u \mid x)\right)+u\right) f(y \mid x) d y\right\|} \\
= & \frac{h_{n}^{\lambda_{1}}}{\left\|D_{1} \theta_{n}\right\|+o\left(\left\|\theta_{n}\right\|\right)} \leq \frac{C h_{n}^{\lambda_{1}}}{\left\|\theta_{n}\right\|} \leq \frac{C h_{n}^{\lambda_{1}}}{n^{-\alpha}},
\end{aligned}
$$

where the first inequality results from the positive definite matrix $D_{1}$ and the second from the definition of $B_{n}$. From (A.13), we know that $h_{n}^{\lambda_{1}} / n^{-\alpha} \rightarrow 0$. Again noting that (A.16), (A.18) and(A.7), then we can see that (A.15) holds. Under Condition 2, by a slight adjustment of the proof of Lemma 5.3 of Chaudhuri (1992), the corresponding results hold analogously for the conditional expectation relating to the variable $\theta$ on the right of the equivalent relationships (A.15). Hence, for all $n$ sufficiently large, there exists some constant $q>0$ such that

$$
\frac{\left\|\mathbb{E} K\left(\frac{x-X}{h_{n}}\right)(U(Y-\theta-Q(u \mid x))+u)\right\|}{\mathbb{E} K\left(\frac{x-X}{h_{n}}\right)} \geq q t K_{2}\left(\log n /\left(n h_{n}^{s}\right)\right)^{1 / 2}
$$

holds for all $\theta$ in $B_{n}$ and $\|\theta\| \geq t K_{2}\left(\log n /\left(n h_{n}^{s}\right)\right)^{1 / 2}$ where $t>0$ is a real. Combination of this and (A.6) results in

$$
\begin{aligned}
& \min _{\substack{\theta \in B_{n} \\
\|\theta\| \geq t K_{2}\left(\log n /\left(n h_{n}^{s}\right)\right)^{1 / 2}}}\left\|\frac{1}{n \mathbb{E} K\left(\frac{x-X}{h_{n}}\right)} \sum_{i=1}^{n} K\left(\frac{x-X_{i}}{h_{n}}\right)\left(U\left(Y_{i}-\theta-Q(u \mid x)\right)+u\right)\right\| \\
& \geq \quad(q t-1) K_{2}\left(\frac{\log n}{n h_{n}^{s}}\right)^{\frac{1}{2}} .
\end{aligned}
$$


By choosing a suitable $t$ such that $q t>1$ and a constant $K_{4} \geq t K_{2}$, Lemma A.7 holds from (A.19), (A.14), (A.13) and the triangle inequality.

According to Lemma A.7, in the sequel, we redefine $B_{n}$ under the further restriction that the norm of each element in it is less than $K_{4}\left(\log n /\left(n h_{n}^{s}\right)\right)^{1 / 2}$. For simplicity, we introduce the notation $\Lambda_{n}(\theta)$ as

$$
\begin{aligned}
\Lambda_{n}(\theta)= & \frac{1}{n \mathbb{E} K\left(\frac{x-X}{h_{n}}\right)} \sum_{i=1}^{n}\left[K\left(\frac{x-X_{i}}{h_{n}}\right)\left(U\left(Y_{i}-Q(u \mid x)\right)-U\left(Y_{i}-\theta-Q(u \mid x)\right)\right)\right. \\
& \left.-\mathbb{E} K\left(\frac{x-X_{i}}{h_{n}}\right)\left(U\left(Y_{i}-Q(u \mid x)\right)-U\left(Y_{i}-\theta-Q(u \mid x)\right)\right)\right] .
\end{aligned}
$$

The following lemma addresses the convergence rate of $\Lambda_{n}(\theta)$, which will be applied to prove Theorem 2.1.

Lemma A.8. Assume that Conditions 2 to 4 and (2.1) hold. If $d \geq 3$, it holds almost surely that

$$
\max _{\theta \in B_{n}}\left\|\Lambda_{n}(\theta)\right\|=O\left(\frac{\log n}{n h_{n}^{s}}\right)
$$

if $d=2$, then for any $0<w<1$, it holds with probability one that

$$
\max _{\theta \in B_{n}}\left\|\Lambda_{n}(\theta)\right\|=o\left(\left(\frac{\log n}{n h_{n}^{s}}\right)^{w}\right)
$$

Proof. Denote by

$$
\begin{aligned}
\xi_{n}(\theta \mid x)= & \mathbb{E}[(U(Y-Q(u \mid x))-U(Y-\theta-Q(u \mid x))) \\
& \left.(U(Y-Q(u \mid x))-U(Y-\theta-Q(u \mid x)))^{T} \mid X=x\right] .
\end{aligned}
$$

For simplicity, given a matrix $A, \operatorname{diag}(A)$ denotes a diagonal matrix the diagonal elements of which is the same as $A$. For any $\theta$ in $B_{n}$ and sufficiently large $n$ it holds that

$$
\leq \frac{\left\|\operatorname{diag}\left(\operatorname{Var}\left(\Lambda_{n}(\theta)\right)\right)\right\|}{\left(n \mathbb{E} K\left(\frac{x-X}{h_{n}}\right)\right)^{2}}\left(\int K^{2}(t) d t g(x)\left\|\xi_{n}(\theta \mid x)\right\|+O\left(h_{n}^{\lambda_{1}}\right)\right),
$$

where the proof is similar to (A.15) and based on the comparison of the convergence rates between $\left\|\xi_{n}(\theta \mid x)\right\|$ and $h_{n}^{\lambda_{1}}$. Applying Lemma 5.7 of Chaudhuri (1992), for $d \geq 3$, it holds that $\left\|\operatorname{diag}\left(\operatorname{Var}\left(\Lambda_{n}(\theta)\right)\right)\right\|=O\left(\log n /\left(n h_{n}^{s}\right)^{2}\right)$ and for $d=2$, it holds that $\left\|\operatorname{diag}\left(\operatorname{Var}\left(\Lambda_{n}(\theta)\right)\right)\right\|$ $=o\left((\log n)^{w} /\left(n h_{n}^{s}\right)^{1+w}\right)$. From these, recalling Fact A.1, Borel-Cantelli lemma and the proof of Lemma A.3, we can obtain (A.20) and (A.21) similarly. 
For any $\theta \in \mathbb{R}^{d}$, we introduce two notations respectively as

$$
\begin{aligned}
\Delta_{n}(\theta)= & \frac{1}{\mathbb{E} K\left(\frac{x-X}{h_{n}}\right)}\left\{-\mathbb{E} K\left(\frac{x-X}{h_{n}}\right) U(Y-Q(u \mid x))\right. \\
& \left.+\mathbb{E} K\left(\frac{x-X}{h_{n}}\right) U(Y-\theta-Q(u \mid x))+\mathbb{E}\left[K\left(\frac{x-X}{h_{n}}\right) P(Y-Q(u \mid x))\right] \theta\right\}
\end{aligned}
$$

and

$$
\begin{aligned}
\Delta_{n}(\theta \mid x) & =-\mathbb{E}[U(Y-Q(u \mid x)) \mid X=x]+\mathbb{E}[U(Y-\theta-Q(u \mid x)) \mid X=x]+D_{1} \theta \\
& =\mathbb{E}[U(Y-\theta-Q(u \mid x))+u \mid X=x]+D_{1} \theta
\end{aligned}
$$

where $D_{1}$ is already defined in Theorem 2.1 .

Lemma A.9. Assume that Conditions 1 to 4 hold. For $d \geq 3$, it holds that

$$
\sup _{\|\theta\| \leq K_{4} \sqrt{\log n / n h_{n}^{s}}}\left\|\Delta_{n}(\theta)\right\|=O\left(\frac{\log n}{n h_{n}^{s}}\right) .
$$

For $d=2$, it holds that

$$
\sup _{\|\theta\| \leq K_{4} \sqrt{\log n / n h_{n}^{s}}}\left\|\Delta_{n}(\theta)\right\|=o\left(\left(\frac{\log n}{n h_{n}^{s}}\right)^{\frac{1+w}{2}}\right) .
$$

Proof. Analogous to the previous proof of (A.15), it is not difficult to show that

$$
\left\|\Delta_{n}(\theta)-\Delta_{n}(\theta \mid x)\right\| \leq C h_{n}^{\min \left\{\lambda_{1}, \lambda_{2}\right\}}
$$

holds for sufficiently large $n$ and any $\theta \in B_{n}$. By Taylor's expansion, (A.17) and Lemma 5.3 of Chaudhuri (1992) in the case of conditional probability measure, for any $\theta \in R^{d}$, there exists constant $0<\eta<1$ such that

$$
\mathbb{E}[U(Y-\theta-Q(u \mid x)+u) \mid X=x]=-\mathbb{E}[P(Y-\eta \theta-Q(u \mid x)) \mid X=x] \theta^{T} .
$$

According to the same argument of Fact 5.8 of Chaudhuri (1992) and using his Lemma 5.7 in the case of conditional probability measure, we can derive that (A.22) and (A.23) hold when $\Delta_{n}(\theta)$ is substituted by $\Delta_{n}(\theta \mid x)$ in these two expressions. Then, in view of (A.24) and Condition 4, both (A.22) and (A.23) hold.

In view of Lemma A.6 and (A.13), the following Lemma 10 can be obtained by the similar argument as the previous Lemma A.3.

Lemma A.10. Under Conditions 1, 2, 3 and 4, it holds with probability one that

$$
\left\|\sum_{i=1}^{n} w_{n, i}\left(U\left(Y_{i}-Q(u \mid x)\right)+u\right)\right\|=O\left(\sqrt{\frac{\log n}{n h_{n}^{s}}}\right) .
$$




\section{A.2 Proof of Theorem 2.1}

Proof. Recalling Lemmas A.6 and A.10, we get that

$$
\left\|\sum_{i=1}^{n} w_{n, i}\left(U\left(Y_{i}-Q(u \mid x)\right)+u\right)\left(\frac{\sum_{j=1}^{n} K\left(\frac{x-X_{j}}{h_{n}}\right)}{n \mathbb{E} K\left(\frac{x-X}{h_{n}}\right)}-1\right)\right\|=O\left(\frac{\log n}{n h_{n}^{s}}\right) .
$$

Similar to the proof of (A.15), it can be shown that

$$
\left\|\frac{\mathbb{E} K\left(\frac{x-X}{h_{n}}\right) P(Y-Q(u \mid x))}{\mathbb{E} K\left(\frac{x-X}{h_{n}}\right)}-\mathbb{E}[P(Y-Q(u \mid x)) \mid X=x]\right\|=O\left(h_{n}^{\min \left\{\lambda_{1}, \lambda_{2}\right\}}\right) .
$$

Accordingly, by (A.13) and Lemma A.7, it holds that

$$
\left\|\left(\frac{\mathbb{E} K\left(\frac{x-X}{h_{n}}\right) P(Y-Q(u \mid x))}{\mathbb{E} K\left(\frac{x-X}{h_{n}}\right)}-\mathbb{E}[P(Y-Q(u \mid x)) \mid X=x]\right) \theta_{n}^{*}\right\|=O\left(\frac{\log n}{n h_{n}^{s}}\right) .
$$

Also, from the definitions above, we have the following relationships

$$
\begin{aligned}
& \sum_{i=1}^{n} w_{n, i}\left(U\left(Y_{i}-Q(u \mid x)\right)+u\right) \\
= & \Lambda_{n}\left(\theta_{n}^{*}\right)+\frac{\sum_{i=1}^{n} K\left(\frac{x-X_{i}}{h_{n}}\right)\left(U\left(Y_{i}-\theta_{n}^{*}-Q(u \mid x)\right)+u\right)}{n \mathbb{E} K\left(\frac{x-X}{h_{n}}\right)}-\Delta_{n}\left(\theta_{n}^{*}\right) \\
& +\left(\frac{\mathbb{E} K\left(\frac{x-X}{h_{n}}\right) P(Y-Q(u \mid x))}{\mathbb{E} K\left(\frac{x-X}{h_{n}}\right)}-\mathbb{E}[P(Y-Q(u \mid x)) \mid X=x]\right) \theta_{n}^{*} \\
& -\sum_{i=1}^{n} w_{n, i}\left(U\left(Y_{i}-Q(u \mid x)\right)+u\right)\left(\frac{\sum_{j=1}^{n} K\left(\frac{x-X_{j}}{h_{n}}\right)}{n \mathbb{E} K\left(\frac{x-X}{h_{n}}\right)}-1\right) \\
& +\mathbb{E}[P(Y-Q(u \mid x)) \mid X=x] \theta_{n}^{*} .
\end{aligned}
$$

Accordingly, from this, Lemma A.8, Lemma A.9, (A.14), (A.25), (A.27), (A.13) and

$$
\left\|\theta_{n}^{*}-\hat{Q}_{n}(u \mid x)+Q(u \mid x)\right\|=O\left(n^{-\alpha}\right),
$$

we know that Theorem 2.1 holds.

\section{References}

Abdous, B., and Theodorescu, R. (1992), "Note on the spatial quantile of a random vector", Statistics \& Probability Letters, 13, 333-336. 
Bosq, D., and Lecoutre, J.P. (1987). Théorie de l'estimation fonctionnelle. Economica.

Cadre, B., and Gannoun, A. (2000), "Asymptotic normality of consistent estimate of the conditional L1-median", Annales de l'I.S.U.P., 44, 13-35.

Chakraborty, B. (2003), "On multivariate quantile regression", Journal of statistical planning and inference, 110, 109-132.

Chaudhuri, P. (1992), "Multivariate location estimation using extension of R-estimates through U-statistics type approach", The Annals of Statistics, 20, 897-916.

Chaudhuri, P. (1996), "On a geometric notation of quantiles for multivariate data", Journal of the American Statistical Association, 91, 862-872.

De Gooijer, J.G., Gannoun, A., and Zerom, D. (2002). "A multivariate quantile predictor". Submitted for publication.

Devroye, L. (1981), "On the almost everywhere convergence of nonparametric regression function estimates", The Annals of Statistics, 9, 1310-1319.

Kemperman, J.H.B. (1987). The median of a finite measure on a Banach space, in Statistical Data Analysis Based on the $L_{1}$ Norm and Related Methods, ed. Y. Dodge, Amsterdam: NorthHolland, pp.217-230.

Serfling, R.J. (1980). Approximation Theorems of Mathematical Statistics. Wiley Series in Probability and Mathematical Statistics.

Serfling, R.J. (2002), "Quantile functions for multivariate analysis: approaches and applications", Statistica Neerlandica, 56, 214-232. 